In gleicher Richtung ist neuerdings auch Martin für die Befreiung des Uterus und der Adnexa aus pelvi-peritonitischen Verwachsungen von der Scheide her, durch die "Vaginale Uterusexstirpation" eingetreten.

Zweifelhaft kann die Berechtigung der vaginalen Hystero-Salpingo-Oophorektomie sein für Fälle von Hydro- und Hämato-salpinx; doch habe ich ihr auch hier stets den Vor\%ug gegeben, namentlich wenn gleichzeitig funktionelle und anatomische Störungen der Gebärmutter, sowie Ueberbleibsel von Pelvi-peritonitis mit absoluter Sterilität vorhanden waren. Auf diesen Errwägungen und Erfahrungen fussend habe ich 100 vaginale Hrsterektomieen mit glitcklichem Ausgang vollbracht. Iie Fälle sind nicht ausgewählt; es sind auch Operationen einbegriffen, die ich schon vor Jahren ausführte, als ich noch Assistent an der Turiner Klinik unter Leitung ron Professor 'T ibone war. Ich habe die Fälle in einer kurzen Uebersicht zusammengestellt.

In einer demnächst erscheinenden Arbeit werde ich die Fälle ausführlicher besprechen und weitere Resultate veröffentlichen.

Uebersicht:

1. Bðrsartige Geschwülste der Gebärmutter . . . . . 23 Fälle.

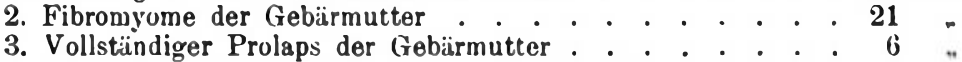

4. Metro-Salpingitis mit Pelvi-peritonitis tuberculosa . . . . 3

5. Schrumpfende Parametritis . . . . . . . . . 1 Fall.

6. Abgekapselte Hämatocele mit doppelseitiger SalpingoOophoritis . . . . . . . . . . . . . 2 Fälle.

7. Entzündungen der Anbänge nit Fiterung . . . . . . . . 20

8. Entzündungen der Gebärmutter mit doppelseitiger Hämato-

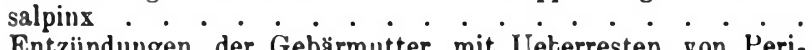

9. Entzündungen der Gebärmutter mit Ueterresten von Yerimetritis, doppelseitiger Hydrosalpinx, Oophoritis oder kleineren

10. Chronische Metritis bei Frauen, welche durch ein- oder doppelseitige Salpingo-Oophorektomie bereits auf abdominalem Wege operiert wurden .

Kein Todesfall.

Zusammen 100 Fälle.

(Aus der Dr. Czempin schen Privatfrauenklinik Berlin.)

\title{
VII. Zur Casuistik der Dermoidcysten des Ovarium
}

Von

\author{
Dr. E. M. SIMONS, \\ I. Assistenten der Klinik.
}

Sowohl klinische, wie pathologisch-anatomische Besonderheiten lassen die Veröffentlichung des folgenden kasuistischen Beitrages als berechtigt erscheinen, um so mehr als einerseits ïber die Aeti- 
vlogie und Nosologie der ovariellen I)ermoideysten und ihrer Abarten (Teratome) die Akten noch keineswegs geschlossen sind und andererseits in klinischer, vor allem diagnostischer Beziehung das Thema dieser C'ysten dauernd zur Diskussion steht.

Fräuloin F., 28 Jabre, litt längere Zeit an starker Rachitis, lernte erst mit $2^{1 / 2}$ Jahren gehen, entwickelte sich geistig sehr spät. Menstruiert seit dem 13. Jahre, anfangs 3 wöchentlicb, spiiter $4-5$ worctentlich, măssig stark, 2 tăgig, nicht sehr schmerzhaft. Nullipara. Niemals hat Scheidenfluor bestanden, ebensowenig litt sie an Ürin- oder Stublbeschwerden. Ihre jetzigen Beschwerden, anfallsweise, in ca. 8 tärigen Pausen auftretende, $2-3$ Tage daulernde, krampfartige Unterleibs- und Kreuzschmer\%en, verbunden mit Schivindolgefühl und Uebelkeit datiert Patientin seit 4 Monaten, da sie einen Sturz von der Leiter gethan hatte. Seit ca. j̇ Jahren aber leidet sie büutig an krampfarsigen Leibschmerzen, die vom Hausarzt immer auf Mlagen oder Darm bezogen wurden. Seit längerer Zeit hat sie auch Anscbwellung des Leibes bemerkt. Der Hausarzt konstatierte jetzt eine bestehende schwangerschaft, obschon die Muglichkeit einer solchen auf das Allerentschiedenste bestritten wurde.

Status: Schlanke, magrere IDunkelblondine mit etwas leidendem Gesichtsausdruck. Knochen sehr gracil, aber nirgends verkrümmt. Stirnschädel etwas vorgebaut. Hals - und Brustorgano ergeben keine klinische Besonderheiten. Abdomen gespannt, entspreclıend der Grosse bei einer 6 MLunate Schwangeren, nur in der Regio epigastrica rechts etwas druckempindlich. Bei gestreckten und geschlossenen sichenkeln ist ein grosses Stück der Rima vulvae sichtbar. Genitaluntersuchung bei der ersten Vorstellung in der Klinik ergiebt einen mannskopfgrossen, im kleinen Becken tief eingekeilten Ovarialtumor, nach oben etwa drei Finger breit den Nabel überragend. Nähere Details bleiben wegen der straffen Bauchdecken und iler Virginität der Scheide in suspenso. In Chloroformnarkose wird kurze \%eit später dieser Befund bestätigt. Der Uterus vorn rechts kaum beweglich. Sondenlange: $7 \mathrm{~cm}$. Hinter ihm, vom linken Parimetrium ausgehend und anscheinend innerbalb des Ligamentes bis tief auf den Beckenboden reichend, eine Hluktuierende, derbwandige. eysti.sche Geschwulst, die, nach oben sehr ver-

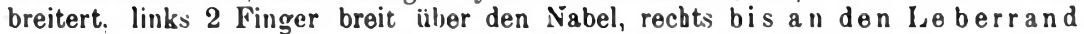
reicht, unbeweglich; die vordere obere Partie fïhlt sich kleinhückerig an. Percussionsschall iaberall gleichmässig: gediimpft-tymphanitisch. Wahrscheinlichkeitsdiagnose lautet: Kystoma multiloculare permagnum. Von einer Probepunktion wird Abstand genommen.

Operation 12. Oktober 189.). Chloroformnarkuse, später Bill roth sche Mischung. Ziomlich ergiebiger Bauchschnitt. Das schwartig verdickte Peritoneum parietrle, das Netz und die Serosa der Cystenwand sind innig miteinander verschmolzen und nicht einzeln differenzierbar. Bei dem Versuche, bier stumpf einzudringen, bricht die noch nicht vollig freigelegte Cystenwand an ibrem obersten Pole ein. und aus der Wunde fliesst mit starkem I rucke eine dünne, in der Farbe schlechtem (Eiter ahnliche Fliissigkeit, ${ }^{1}$ ) so dass das ganze Bild zunächst den Eindruck einer tuberkulosen ['eritonitis und Pseudatumor macht. Nach links von 1er Einbruchsstelle ist die Bauchhühle eröffnet. Die weisslich-gelbliche Flüssigkeit wird moglichst nach aussen geleitet, es kann aber nicht verhindert werden, dass eine betrăchtliche Menge über das Operationsgebiet fliesst. Fis ist auch nicht nuoglich, die Cyste auch nur annähernd gänzlich zu entleeren und so bei den späteren Manipulationen die vielfache Berübrung des Dermoidinhaltes mit den verschiedenen Abschnitten des Peritoneum zu verhindern. Auf allen Gegenstlinden, mit welchen sie in Berührung kommt (Operationstisch, Fussboden, Auffange-Schale), nimmt die Fliussigkeic bald eine feste, talgartige Consistenz an und die Diagnose wird vollends sichergestellt, als Operateur beim Ausschüpfen des Sackes aus ler T'iefo 2 faustgrosse Büscbel blonder Huare hervorhebt.

Der Wundrand des Sackes ist von seiner Umgebung kaum zu differenzieren und $1 \mathrm{~m}$ die Ausschălung des letzteren zu erleichtern, wird derselbe mit Mullservietten lose vollgestopft; dann wird die Cystenmembran an ihrer oberen Finbruchstelle vorsichtig nach allen Seiten stumpf abprïpariert und die ge-

1) Engstr $ð \mathrm{~m}$, Centralbl. 1887, 5, fand ebenfalls hellgelbe wasserdünne Flüssigkeit in einem, nach unten intraligamentären Dermoid. 
wonnene Jembran zur bequemeren Handhalur.g nit Klemmen gefasst. Es zeigt sich nunmehr, dass der ganze obere l'ol der Geschwulst, ferner ihre HinterHiiche bis zum Krouzbeine hinab und ihre linke Seitenwand nit zahlreichen, in chronischer Fntzündung bochroten, unter einander fest verklebten Darmschlingen aut das Innigste verirachsen ist. Ebenso ist das wulstig verdickte Netz mit den Diimen verklebt und sowie an die rordere und rechtsseitige Wand der Crste an das Peritoneum parietale der vorderen Bauchwand breit fixiert. is bildet somit der ganza Inhalt der Bauchboble ein Convolut vollkommen verwachsener Organe. Langsam und sehr vorsichtig werden die l)ärme stumpf an der ausseren Cystenwand abgelost, ohne dass erbebliche Blutungen entstănden. Allerdings sind einige Verwachsungen mit Dünndarmschlingen so fest, dass ganz kleine Partieen der Cysten wand, aus dieser abgetragen, an jenen haften bleiben müssen. Nach rechts ist es inmer noch unnoglich, uber den Verlauf der eruftineten Cystenwand sich zu orientieren. Nach unten, median hinter den Uterus hat der Sack, das hintere Blatt des Ligam. lat. nit rordrängend, sich bis aut den Buden des - Douglas" hinabgesenkt; das beisst, er ist in unteren Teile intraligamentär entwichelt. Nachdem Operateur, mehrfach nit der einen ganzen $\mathrm{Hand}$ in die Cyste eingehend, deren buchtenreiche Wand tixiert und so der aussen auf der Cystenuall arbeitenden anderen Hand die Lðsung der Verwachsungen ermüglicht hat, gelingt es endlich, den Sack nitsant seinem serusen Ueberzug aus den kleinen Becken emporzurollen. An seiner äussersten linken Partie verläuft, Huf den Sacke festsitzend, die gestreckte, tiefrute Tuba. Durch dieses Fmporziehen der Cyste lässt sich ein gut charakterisierbarer Stiel bilden. Un jedoch beim Ablösen nicht ubermissig viel Gewebe des Ligam. lat. mitzumehnen. wird dieser Siriel \%unichst lose in Klemmen gefasst, das die Cyste umgebende Peritoneum aut der hinteren $W$ and eingeschnitten und die eigentliche Cyste aus dem Ligan. lat. ausgelost. Nunnehr lassen sich Ligamer:t. in iundibulo-pelvicun, das breite Iutterband und die Uteruskante bequen ligieren. Der mehrfach zerfetzte. durch die intraligamenthre Entwickelung der Cyste aus dem hintern Blatt des breiten Nutterbandes entstandene Serosasack wird natürlich grひsstenteils reseciert Der grebildete Stiel ist schr breit und bedarf nach Ablösung der Cỵste noch mehrfacher Unterbindungen.

Die Exploration der rechten Adnexe hat gleichfalls mit der stumpien Ablusung \%ahlreicher Jarmadhäsionen zu heginnen, um zunächst überbaupt cine Orientierung zu ermoglichen. Dabei dringt plotelich neben dem Finger des Operateurs in breitenı Strahle grünlich gelber, stinkender Eiter aus dor Tiefo hervor, dessen Findringen in die Bauchbohle nach 1 oglichkeit hintertrieben wird. Derselbe stanmt aus der lidierten $W$ and eines ca. $18 \mathrm{~cm}$ langen, über daumendicken, viernal gewundenen, an einem sehr fleischigen, breiten Mutterbande in cerader Richtung ansitzenden l'ubensackes, der bis in die Hohe des unteren I,eberrandes hinaufreicht und in dieser Gegend mit dem parietalen Bauchfellblatte durch die l'rodukte einer ziemlich jungen "plastischen" Entziindung verwichsen ist. Der Ansatz der Adnexe am Uterus zeigt gewissermassen 2 isolierte Frontralebenen, inden das sehr derbe Ligament. orarii scheinbar einen eignen serosauber\%ug trigt. I)iese Situation ist oftenbar dadurch \%ustande gekummen. dass der 'J'ubensack durch seine mächtige Entrvickelung nach oben den obcrcn land des Ligament. lat. nit weggedrangt und so zwischen ihm und dem Eierstucksbande eine geröumige serðse Tasche gelassen bat. Denentsprechend bedürfen die Adnexe an ihrem uterinen Ende in ihren einzelnen Teilen besonders starker I,igaturen. Anschliessend an diese wird mit starken Seidenfäden das breite .lutterband und das Ligam. infundibulo-pelricum abgebunden und mit kleinen scheerenschlägen abgetragen. Revision ergiebt an den Stiumpfen keine Nach. blutung, wohl aber eine solche, mehr parenchynatosen Charakters gan\% in der 'Tiefe, auf dem Beckenboden, dort, wo die Dermoidcyste ansgegraben worken war. l)iese Gegend wird nit steriler Gaze tamponiert. Abbinden und Resection mehrerer bedeutender Netzzipfel. Schluss der I3auchwände bis zu der für die Herausleitung des Gazetampons bleibenden kleinen Dettinung; vorber aber recoll. struiert sich Operateur durch nachtrăgliche deutlicae Isolierung das entziundlich adharente l'eritoneum parietale. Eine isolierte Naht der einzelnen Bauchschichten, wie sie sonst in der Klinik üblich ist, ist wegen der langen Dauer der (Operation schwer angăngig, weshalb durchgreifende Silkwormnihte gewahlt werden. Dauer der Operation $2^{1 / 4}$ Stunden; Narkose ungestort. 
In klinischer Hinsicht ist in erster I inie interessant lie Schwierigkeit der I)iagnose. Ier bekannte Symptomenkomplex der pläuterinen lage einer, passir weggedrängt, immer wieder in ihre Lage „Zurïckschnellenden“ (Geschwulst, das sogenannte $\mathrm{K}$ ï s ter sche Zeichen, dessen pathognomonische Bedeutung iibrigens ron Lat $\% \mathrm{ko}$ in jüngster Zeit wohl mit Recht abermals in Abrede gestellt wird, kam überhaupt nicht in Betracht, da es sich - dies war bald zu cruieren - um eine, zum 'Teil intraligamentär entwickelte, und schon deshalb unbeweglich hinter dem Uterus liegende Cyste handelte. Das von Kocher (Centralblatt f. Chirurgie 1887) bei der Palpation von Iermoidcysten konstatierte Reiben der Haare - als Beobachtung vermutlich eine grosse Seltenheit - war nicht vorhanden. Das wegen seiner Gefährlichkeit schon in wohlberechtigten Misskredit geratene Hilfsmittel der Probepunktion wurde abgelehnt, weil es -- kein Hilfsmittel ist. Angenommen in unserem Falle hätte die Probespritze den Befund: Iermoidinhalt ergeben, - - wiiren uns etwa die bei der Operation die betreffenden Ueberraschungen erspart geblieben? Wăre man aber gar mit der Spritze zufällig in die Pyosalpinx geraten, so hätte dieser diagnostische Hinweis sogal in falschester Wrise gedentet werden mitssen. Um in einem betreffienden Falle die Indikation auf luaparotomie stellen $\%$ können, wird man überhaupt meist solcher Hilfsmittel entraten und sich auf das Ergebnis einer Narkosenuntersuchung allein stiot\%en können. In unserem Falle konnte allerdings nicht einmal in (hloroformnarkose auch nur das äussere Verhalten der Geschwulst, ihr Verhältnis \% den Nachbarorganen, speciell zu den anderseitigen Adnexen eruiert werden. Auch die von Schultze angegebene Methode, den mit einer Hakenzange gefassten Uterus herab\%uziehen, gewahrte keine Klairung, obschon das Organ sich etwas nach abwarts bewegen liess. Das Conglomerat der auf einer grossen Fläche beider Säcke kreuz und ruer festgewachsenen I)armschlingen liess eben selbst durch die in Narkose röllig erschlaftiten Bauchdecken hindurch Dermoidsack plus Eileitersack als cine ein rige Orarialcysste und zwar mit grösster $W$ ahrscheinlichkeit als multilokuläres Cystom erscheinen.

In der Sit\%ung der Gesellschaft fur Geburtshilfe \%u leipzig, 20. Oktober 1884 demonstrierte Sianger einen Fall ron Orarialcyste, die in ganz ähnlicher Weise wie die unserige mit Darmschlingen röllig iberdeckt war, sodass die Cyste \%wischen den Därmen musste herausgeschält werden. Sänger deutete auch auf die diagnostische l,ehre hin, die man aus dieser pathologischen Anordnung zu ziehen habe.

Aber sogar bei der Operation, nach Erötinung des Bauches und selbst des Sackes - verhinderten in unserem Falle die Schwierigkeit der Uebersicht sowie die eigenartige Veränderung der Dermoidflissigkeit eine Diagnosenstellung, bis die beiden Haarbisschel entfernt wurden.

Bei der anscheinend bestehenden Seltenheit von gleichzeitigem Vorkommen einer Ovarialcyste einerseits und einer Hitertube andererseits, so zwar, dass beide zu gleichwertigen Krankheits- 
depots werden konnten, - die letzten 'Tage ror der Operation hatte gerade die Pyosalpinx akut peritonitische Irscheinungen rerursacht, - liegt die Frage nahe, ob es sich um eine zufallige Coïnciden\% oder irgend einen Causalnexus handele. Fiar letzteren sprechen schu'erwiegende Gründe.

Dass die rechtsseitige 'T'ubenerkrankung eine autochthone sein könne, ist fürs erste kaum möglich, weil bestimmt niemals filuor e genitalibus bestanden hat. Der Connex zwischen den verschiedenartigen beiderseitigen Adnexerkrankungen dürfte vielmehr folgender sein: wie so oft verursachte die wachsende Dermoidcyste Peritonitis in der äusseren Lmgebung des Sackes, also auch in der Serosa der benachbarten rechtsseitigen Tube. - Als Beweis hierfür gelten die zahlreichen Tuben-Darmverwachsungen, als Folgeerscheinung resultierte Salpingitis und Hydrosalpinx, dessen Inhalt durch das sklerosierte uterine Ostium in den Cterus abzuführen die T'ubenwiinde immer wieder, und \%war auch deshalb vergebens versuchten, weil die 'Tube in der von Freund beschriebenen Weise (foetale Entwickelungshemmung), einen mehrfach gewundenen Schlauch darstellte. Vielleicht sind die oben erwähnten, zeitlich lange zuriickliegenden "Krämpfe" als der klinische Ausdruck für solche Auspressversuche auf\%ufassen, wiahrend als pathologisch-anatomische Folgeerscheinung aus derselben (Quelle die Arbeitshypertrophie der 'Tlibenwand, im Sinne sowohl des I)icken- wie des liängenwachstums resultierte.

Das auffallende Missverhältnis zwischen dem sehr grossen 'Tubensack und der schlecht entwickelten Gebiirmutter, die, der Regel \%utrotz, keineswegs mit jenem zugleich in die Länge gewachsen, sondern nur in toto etwas hochgezerrt war, scheint ebenfalls die Ableitung des Sekretes nach aussen erschwert \%u haben.

Aus der chronischen Hydrosalpinx ist dann wohl die l'yosalpinx durch Einwanderung des vielgewandten Bacterium coli commune aus den (lauernd so intim benachbarten, \%ahlreichen, adliaerenten Darmschlingen entstanden. I)ie Annahme dieser Aetiologie erscheint voll berechtigt. denn Gonorrhoe und Endometritis fehlen völlig in der Anamnese, des weiteren aber bewies der giinstige Verlauf des Falles die nicht septische Beschattenheit des, doch mit den rerschiedensten Serosapartien in Berührung gekommenen 'Tubeneiters.

Ebenso harmlos dem P'eritoneum gegenüber hat sich übrigens die ausgetretene Dermoidflüssigkeit gezeigt, wieder eine Bestätigung der zuerst ron Engström (Centralblatt 1887, No. 5) bewiesenen Thatsache, dass die firiher allgemein verbreitete grosse Aengstlichkeit vor der bedeutenden Infectiosität des Dermoidinhaltes nicht gerechtfertigt ist.

'/ur Darlegung der pathologisch-anatomischen Besonderheiten unseres Falles mag die Beschreibung des Priiparates folgen.

In wenig geschrumpftem Zustande (Formalin) hat dic entleerte Cyste eino Lïnge von 17 und eine grosste l3reite von $12 \mathrm{~cm}$. Ihr äusserer Ueberzug ist durchaus seriser Natur. Die Dicke der Wand variiert bedeutend, von $1 \mathrm{~mm}$ bis $1 \mathrm{~cm}$. Panniculus adiposus feblt vollig. Der Innenraum besteht aus einer kleineren und einer grösseren Kanmer, welch letztere zum weitaus grössten 'l'eile mit echter, derber Haut ausgekleidet ist, über der zahlreiche, nit Fett oder 
Talg vermischte Epithelien liegen. An einzelnen, inselartig schart umyrenzten Partien fehlt die Epidermis gînzlich, und es schimmert die glatte Aussenfliche der Cyste rotlich durch die gleichformige Wand. ${ }^{1}$ ) In der dicken, mit grossen Haarfollikeln ausgestatteten Haut sitzen zahlreiche blonde Haare fest. andere liegen unter einander verklebt, frei im Cystenlumen. Die unvollständige Abgrenzung der unteren grosseren ron der kleineren oberen Kanmer geschieht durch eine melirfach geschweifte, sehr derbe Leiste, die, wie durch eine ursprüngliche Wandeinstiilpung in das Lumen hinein entstanden, sich quer durch den ganzen sack spannt. etwa in der Mitte eine cylindrig ausgezogene Spitze nach der gregenuberliegenden C'ystenwand sendet. $31 \% \mathrm{~cm}$ von dieser Spange entfernt ragt aus der Leiste ein Kahn empor mit Krone, ohne Email; derselbe sitzt fest auf einem $5 \mathrm{~cm}$ langen. nach Wegpriparieren der Haut erst deutlich werdenden: platten Kinochenstucke, welches wie ein Unterkiefer gebogen ist. $2 \mathrm{~cm}$ oberLalb dieser Knochenleisto drangt sich die bieretwas zartere lipidermiswand in tlachen Wulste vor. Nach vorsichtigen Abpräparieren der Haut zeigt sich, dem Wulst entsprechend, ringsberum deutlich differen\%iert, in lockeres Bindegewebe eingebettet, ein $3^{1,2} \mathrm{~cm}$ langer, 2 cn breiter, un regelmassig gelappter. drisenartiger Korper, der in Form. Farbe und Consistenz sofort an eine 'arotisdrüse erinnert.

I)ie mikroskopische Untersuchung der Driise bestätigt die Richtigkeit des Vergleiches mit einer (älandula parotis. Härtung in Alkohol. Celloidineinbettung, Alaun-Carmin-Färbung. Die Schnitte fallen meist senkrecht zu der lä̈ngsrichtung der 'Tubuli und geben das typische Bild der in lockeres Bindegewebe mosaikartig eingelegten Schläuche. Die grossen, trapezoid gestalteten Epithelien, die nur selten das central gelegene Kanallumen erkennen lassen, tragen ihren breiten Kern meist mehr peripher und zeigen niemals hellen, homogenen, sondern auschliesslich feinkörnigen Protoplasmainlialt. Es sei dies besonders betont, weil in den später zu erwähnenden, aus der I itteratur zusammengestellten Fällen mit ähnlichen Befunden meist von Schleimdriisen die Rede ist. Unsere Iriise hat sicherlich kein mucinhaltiges, sondern ausschliesslich seröses resp. Hiweisssekret geliefert, also auch in physiologischer Hinsicht das Beispiel der menschlichen Ohrspeicheldruse durchaus imitiert. Im mikroskopischen Bilde fehlen auch nicht die sogenannten „D)riisenschaltsticke“ mit ihrem unregelmässigen, etwas plattgedrïckten Epithel. Nach den charakteristischen speichelgiingen mit ihrem ,feingestrichelten“ Cylinderepithel habe ich allerdings rergeblich gesucht, wie auch die grossen, mehrfach gewundenen Sammelröhren nicht cinfaches Cylinderepithel, sondern mehrschichtiges Epithel aufweisen, dessen basale Reihe an einzelnen, stark gewundenen Stellen den cylindrischen 'Typus annimmt. An einzelnen, besonders erweiterten Partieen dieser Kanile sieht man das, jedenfalls gestaute Sekret.

Eine dermassen deutlich isolierte, grössere „acinöse“ oder besser . \%usammengeset $\% t$ tubulöse" Drüise in einem Ovarial-I)ermoide finde ich in der litteratur nicht beschrieben.

In der Sitzung der Berliner Gesellschaft für Geb. u. Gynăk., 10. Jan. 1890, demonstrierte Fla ischlen einen ron P. Ruge

1) Poupinel (citiert von Pozzi in soinem Lehrbucbe) fand ebentalls das Innere einer Dermoidcyste ausgekleidet teilweise mit echter Haut, mit allen ihren Luthaten, teilweise gleichformig glatt. 
durch Laparotomie entfernten Dermoidtumor des Ovarium, eine Combinationsgeschwulst teils colloiden, teils dermoiden Inhaltes, in welcher el ausser den gewöhnlichen Attributen dieser Cysten einen drïsenartigen Körper fand ron Form und Grösse einer Submaxillardriise, ohme jedoch in dem, mit Detritus durchsetzten Stiitzrewebe, welches dem.jenigen einer ,acinösen“ Driise entsprach, „ordentlich erhaltene epitheliale Elemente* entdecken \%u können.

Keller, Centralbl. 1888, s. 75.2, fand in einem, nach allen Richtungen hin untersuchten, an Funden der versehiedensten Gewebsarten reichen Falle koine Spoicheldrüsen; wohingegen Böttlein in seinem interessanten Falle (Virch. Arch. 1889, CXV, pag. 493) neben einer cigentiimlichen Höhle ,ziemlich dichtstchende Massen“ wahrnahm, „die die grösste Achnlichkeit mit acinosen Schleimuriisen und Hiweiss" resp. "serösen Drilsen“ hatten.

Fribher bereits hatte auch Baumgarten etwas Aehnliches gesehen (Virch. Arch. CVIl, s. jıj u. l216), nämlich „acinose“ schleimdriisen, die ihr Seklet in kleine Schleimcystchen abgaben, sodass ,die Präparate nicht greringe Achnlichkeit mit Schnitten duch embryonale Magen- und Darmwand aufwiesen." Iieselben Verhaltnisse konstatierte Marchand (citiert bei ()lshausen: Krankheiten der (Orarien).

E'mannel, 'Teratoma orarii, Zeitschril't fïr Gebh. und Gynaek. Band 25, erwähnt ebenfalls acinöse I)rüsen, dic seinen, der Abhandlung beigegebenen mikroskopischem Bilde nach aber mit Speicheldriisen gral keine Achnlichkeit hatien.

Unsere grut difterenzierte und solitäre Driise wies also makroskopisch und mikroskopisch dic Charaktere einer echten Eiweisssekret-I)riise auf, deren plyysiologrisches l'rototyp wir in der menschlichen ()hrspeicheldrise kennen. Es erscheint mir laher der Hinwris erlaubt, auf solche Hischeinungen in den Dermoiden, mehr als es bisher geschehen ist, zu achten, ja, crentuell rielleicht in lem Crsteninhalte nach den chemischen Attributen les Siekretes dicser I) riisen (Ptyaliu, Rhodankalium), sowie nach den bekannten Speichelkürperchen \% fahnden.

Noch einige Worte über len klinischen Verlau f unseres Falles. Abends nach ler Operation 'T'mperatur 38.5) (am Abend rorher 38). Am \% weiten 'Tage 38,7 abends, bis \%um vierten Tage Ablall zur Norm; subjektives Betinden gut. Am vierten 'lage Verbandwechsel mit Entfermung der (iaze ans der Banchhöhle und 1)ränage des Wundtrichters. Im laute del folgenden Wochen schliesst sich let\%terer unter mehr weniger starker Eiterung bis einer fistulösen ()effinung. Die ubrige Bauchmalit heilt per prim. int. Die Rekonralescenz ist ctwas rer\%ögert durch eine unerhebliche 'Thrombose der rechten Cruralrene mit begleitenden Fiebererscheinungen bis über 39 abends; anschliessend daran tritt cin durch die Bauchdecken jalpabeles Beckenbindegewebs-exsudat in die Eischeinung. Appetit und Stuhl damernd gut; subjektive Beschwerden sehr gering. $6^{1 / 2}$ IVochen post. operat. steht Patientin zum ersten Male auf. 
Am 20. Dec. 95 rerliess Patientin die Anstalt. Allgemeinbefinden gut. Wenig secernierende Fistel in unteren Wrundwinkel.

Zuun Schlusse erfülle ich die angenehme Pflicht, meinem rerehrten Chef, Herrn I)r. C zemp in, für die Ceberlassung des Falles meinen aufrichtigen Dank \%u sagen.

\title{
Referate und Berichte.
}

\section{Die moderne Eklampsiebehandlung in den Vereinigten Staaten ron Nordamerika}

\author{
Von \\ Ir. FRANY T. B. FEST \\ (Plank Road. L. S. A.)
}

In der nordarnerikanischen Fachlitteratur dreht sich die Frage der Eklampsiebehandlung hauptsächlich um drei Methoden: Venaesektion, Narkose und Veratrum viride. Ceberall jedoch fallt uns ein beliebtes Stichwort der modernen amerikanischen Medicin auf „Fli. mination".

Seit langem schon stand die I)arreichung von Veratrum viride in den Suldstaaten in hohem Ansehen; die guinstigen Berichte erwarben dieser Methode zahlreiche Anhänger, so dass sie seit einer Reihe von Jahren allgemein umstritten wurde. Veratrum viride wurde in der Folge hiervon wohl das beliebteste Medikament der Eklampsiebehandlung, wennschon natürlich Gegner auftraten und sich zal:Ireiche Stimmen für andere Methoden erhoben. Bei der Eklampsia gravicarum wird hierzulande so. fortige Entleerung der Gebïrmutter allgemein für notwendig erachtet. In Berieliung auf die Aetiologie gilt urämische Intoxikation ineist als causales Iloment, während der , Eklampsie-Bacillus“ verhältnismässig weniz krwähnung findet.

Mckeough (1) berichtet den liall einer zuror rollkommen gesunden Frau, bei der die eklamptischen Anfälle am 10. 'Tage post partum auftraten. $/ / \mathrm{u}$ keiner Zeit liess sich im Harne Eiweiss nachweisen, wohl aber eine Masse amorpher I,ithiate. Vorher hatten sich weder Krämpfe noch hysterische Symptome ger.eigt. I) $\mathrm{IV}^{r} \mathrm{i} t$. (2) sah einen Fall, in den die Konvulsionen vierundzwanzig Stunden post partum begannen, während dieser Zeit nach der Enthindung litt die Patientin ohne jegliche Vorboten an völliger Frblindung. Hariet I. Harrington (3) berichtet den Fall einer Frau, bei der zwei Fntbindungen innerhalb eines Jahres stattfanden mit jedesmaliger Komplikation durch eklamptische Konvulsionen. Page (4) beschrieb einen Fall im VIl. Schwangerschaftsmonat. Nach heftigen Konvulsionen erfolgte spontane Fruihgeburt und die Patientin genas. 